PROCEEDINGS OF THE

AMERICAN MATHEMATICAL SOCIETY

Volume 133, Number 10, Pages 3087-3096

S 0002-9939(05)08111-6

Article electronically published on April 25, 2005

\title{
OPERATOR-VALUED FREE FISHER INFORMATION AND MODULAR FRAMES
}

\author{
BIN MENG, MAOZHENG GUO, AND XIAOHONG CAO
}

(Communicated by David R. Larson)

\begin{abstract}
We introduce the operator-valued free Fisher information for a random variable in an operator-valued noncommutative probability space and point out its relations to the amalgamated freeness. Using M. Frank and D. Larson's modular frame notion we can construct the conjugate variable for an operator-valued semicircle variable with conditional expectation covariance. Then we obtain its free Fisher information and show it is equal to the index of the conditional expectation. At last the conjugate variable with respect to a modular frame operator for a semicircle variable is also constructed.
\end{abstract}

\section{INTRODUCTION AND PRELIMINARIES}

About twenty years ago, Dan Voiculescu introduced a noncommutative probability (or "free probability") theory in the framework of operator algebras. The theory soon became a powerful tool in the study of operator algebras. Especially, its connections with random matrices and the notion of free entropy are very important in the study of von Neumann algebras. We would like to refer the readers to [17] for more details on the basic knowledge on free probability theory.

The key notion in free probability theory is "free" independence (or "freeness") replacing the classical independence in probability theory. Originally a free probability space is a pair $(\mathcal{A}, \tau)$, where $\mathcal{A}$ is a $\mathrm{C}^{*}$ - or von Neumann algebra and $\tau$ is a state. Free independence is defined in terms of a reduced free product relation given by $\tau$. This notion was generalized by Voiculescu and others to an algebra-valued noncommutative probability space where $\tau$ is replaced by a conditional expectation $E_{\mathcal{B}}$ onto a subalgebra $\mathcal{B}$ of $\mathcal{A}$, and freeness is replaced by freeness with amalgamation.

Definition 1 ([18). Let $\mathcal{A}$ be a unital algebra over $\mathbb{C}$, and let $\mathcal{B}$ be a subalgebra of $\mathcal{A}, 1 \in \mathcal{B}$. $E_{\mathcal{B}}: \mathcal{A} \longrightarrow \mathcal{B}$ is a conditional expectation, i.e. a linear map such that $E_{\mathcal{B}}\left(b_{1} a b_{2}\right)=b_{1} E_{\mathcal{B}}(a) b_{2}, E_{\mathcal{B}}(b)=b$, for any $b, b_{1}, b_{2} \in \mathcal{B}, a \in \mathcal{A}$. We call $\left(\mathcal{A}, E_{\mathcal{B}}, \mathcal{B}\right)$ an operator-valued (or $\mathcal{B}$-valued) noncommutative probability space and elements in $\mathcal{A}$ are called $\mathcal{B}$-random variables.

The algebra freely generated by $\mathcal{B}$ and an indeterminate $X$ will be denoted by $\mathcal{B}[X]$. The distribution of $a \in \mathcal{A}$ is a conditional expectation $\mu_{a}: \mathcal{B}[X] \longrightarrow \mathcal{B}, \mu_{a}=$

Received by the editors February 12, 2004 and, in revised form, June 7, 2004 and June 8, 2004. 2000 Mathematics Subject Classification. Primary 46L54, 42C15.

Key words and phrases. Conjugate variable, free Fisher information, Hilbert $C^{*}$-module, modular frame. 
$E_{\mathcal{B}} \circ \tau_{a}$, where $\tau_{a}: \mathcal{B}[X] \longrightarrow \mathcal{A}$ is the unique homomorphism such that $\tau_{a}(b)=b$, for any $b \in \mathcal{B}, \tau_{a}(X)=a$.

Let $\mathcal{B} \subset \mathcal{A}_{i} \subset \mathcal{A}(i \in I)$ be subalgebras. The family $\left(\mathcal{A}_{i}\right)_{i \in I}$ will be called free with amalgamation over $\mathcal{B}$ (or $\mathcal{B}$-free) if $E_{\mathcal{B}}\left(a_{1} a_{2} \cdots a_{n}\right)=0$ whenever $a_{j} \in \mathcal{A}_{i_{j}}$ with $i_{1} \neq i_{2} \neq \cdots \neq i_{n}$ and $E_{\mathcal{B}}\left(a_{j}\right)=0,1 \leq j \leq n$. A sequence $\left\{a_{i}\right\}_{i \in I} \subseteq \mathcal{A}$ will be called free with amalgamation over $\mathcal{B}$ if the family of subalgebras generated by $\left(\mathcal{B} \cup\left\{a_{i}\right\}\right)_{i \in I}$ is $\mathcal{B}$-free.

In this paper we mainly consider $\mathcal{B}$-valued $W^{*}$-noncommutative probability space. That is to say, $\mathcal{A}$ is a von Neumann algebra, $\mathcal{B} \subseteq \mathcal{A}$ is a von Neumann subalgebra and $E_{\mathcal{B}}$ is a normal conditional expectation of $\mathcal{A}$ onto $\mathcal{B}$ (i.e. $E_{\mathcal{B}}$ is a norm 1 projection).

In this paper we always assume that conditional expectations are faithful. To be precise, the conditional expectation $E$ satisfies $E\left(x x^{*}\right)=0$ if and only if $x=0$.

D. Voiculescu provided two versions of free entropy: one is defined by the matricial microstates approach and the other is defined by the infinitesimal microstates approach. This paper is about the latter one. In this approach the free Fisher information measure $\Phi^{*}$ comes first and the free entropy is derived from it. So discussing the free Fisher information is essential in the study of this version of free entropy $([20,21])$. However, in this paper we generalize the free Fisher information to the operator-valued settings and prove it to be closely related to the amalgamated freeness just like the free Fisher information $\Phi^{*}$ is related to freeness (see [20]). But unfortunately, we cannot prove this result in the operator-valued settings following D. Voiculescu's way in 20, because, in general, conditional expectations are not tracial (in the settings of [20], there is always a trace $\tau$ on $\mathcal{A}$, that is, $\tau$ satisfying $\tau\left(a_{1} a_{2}\right)=\tau\left(a_{2} a_{1}\right)$, for all $\left.a_{1}, a_{2} \in \mathcal{A}\right)$. We find that using R. Speicher's combinatorial theory in operator-valued free probability can overcome the difficulty. R. Speicher's theory mainly deals with the relations between moment function and cumulant function (see [13]). He uses $\widehat{k_{\mathcal{B}}}:=\left(k_{\mathcal{B}}^{(n)}\right)_{n \geq 1}$ and $\widehat{E_{\mathcal{B}}}:=\left(E_{\mathcal{B}}^{(n)}\right)$ to denote the moment and cumulant functions induced by $E_{\mathcal{B}}$ respectively where $E_{\mathcal{B}}^{(n)}\left(a_{1} \otimes \cdots \otimes a_{n}\right):=E_{\mathcal{B}}\left(a_{1} \cdots a_{n}\right)$ and $k_{\mathcal{B}}^{(n)}$ is determined by the following recurrence formula (where $E_{\mathcal{B}}^{(0)}=1$ formally):

$$
\begin{aligned}
& E_{\mathcal{B}}^{(n)}\left(a_{1} \otimes \cdots \otimes a_{n}\right) \\
&=\sum_{r=0}^{n-1} \sum_{1<i(1)<i(2)<\cdots<i(r) \leq n} k_{\mathcal{B}}^{(r+1)}\left(a_{1} E_{\mathcal{B}}^{(i(1)-2)}\left(a_{1} \otimes \cdots \otimes a_{i(1)-1}\right)\right. \\
& \otimes a_{i(1)} E_{\mathcal{B}}^{(i(2)-i(1)-1)}\left(a_{i(1)+1} \otimes \cdots \otimes a_{i(2)-1}\right) \\
&\left.\otimes a_{i(2)} \cdots \otimes a_{i(r)} E_{\mathcal{B}}^{(n-i(r))}\left(a_{i(r)+1} \otimes \cdots \otimes a_{n}\right)\right)
\end{aligned}
$$

for all $n \in \mathbb{N}$ and $a_{1}, \cdots, a_{n} \in \mathcal{A}$. It is easy to see $\widehat{E_{\mathcal{B}}}$ and $\widehat{k_{\mathcal{B}}}$ determine each other uniquely by the above recurrence formula. Then in [9], the authors gave an equivalent condition of the amalgamated freeness:

Lemma 2 (9]). Let $\mathcal{B}, \mathcal{C} \supseteq \mathcal{D}$ be two subalgebras in $\mathcal{D}$-valued noncommutative probability space $\left(\mathcal{A}, E_{\mathcal{D}}, \mathcal{D}\right) . \quad E_{\mathcal{B}}, E_{\mathcal{C}}$ are the conditional expectations of $\mathcal{A}$ onto $\mathcal{B}, \mathcal{C}$ respectively and satisfy $E_{\mathcal{D}}=E_{\mathcal{D}} E_{\mathcal{B}}=E_{\mathcal{D}} E_{\mathcal{C}}$. Then $\mathcal{B}$ and $\mathcal{C}$ are $\mathcal{D}$-free if 
and only if

$$
k_{\mathcal{B}}^{(n)}\left(c_{1} \otimes c_{2} b_{2} \otimes \cdots \otimes c_{n} b_{n}\right)=k_{\mathcal{D}}^{(n)}\left(c_{1} \otimes c_{2} E_{\mathcal{D}}\left(b_{2}\right) \otimes \cdots \otimes c_{n} E_{\mathcal{D}}\left(b_{n}\right)\right)
$$

for any $b_{1}, b_{2}, \cdots, b_{n} \in \mathcal{B} ; c_{1}, c_{2}, \cdots, c_{n} \in \mathcal{C}$, where $\left(k_{\mathcal{B}}^{(n)}\right)_{n \geq 1},\left(k_{\mathcal{D}}^{(n)}\right)_{n \geq 1}$ are the cumulant functions induced by $E_{\mathcal{B}}, E_{\mathcal{D}}$ respectively.

In the present paper we will use modular frames to compute the free Fisher information of a semicircular variable. The notion of modular frames is a natural generalization of frames in Hilbert space to Hilbert $C^{*}$-module settings. For a unital $C^{*}$-algebra $\mathcal{D}$, a Hilbert $\mathcal{D}$-module is a linear space and an algebraic $\mathcal{D}$-module $\mathcal{M}$ together with a $\mathcal{D}$-valued inner product $\langle., .\rangle_{\mathcal{D}}$ and complete with respect to the Hilbert $C^{*}$-module norm: $\left\|\langle., .\rangle_{\mathcal{D}}\right\|^{\frac{1}{2}}$. We refer to 8 for more details on Hilbert $C^{*}$-module theory. A sequence $\left\{f_{j}: j \in \mathbb{J}\right\} \subseteq \mathcal{M}$ is said to be a frame if there are real constants $C, D>0$ such that

$$
C\langle x, x\rangle_{\mathcal{D}} \leq \sum_{i}\left\langle x, f_{i}\right\rangle_{\mathcal{D}}\left\langle f_{i}, x\right\rangle_{\mathcal{D}} \leq D\langle x, x\rangle_{\mathcal{D}}, \text { for all } x \in \mathcal{M} .
$$

When $C=D=1$ the frame will be called normalized tight. Note that for a frame the sum in the middle only converges weakly, in general. If the sum converges in norm, then the frame will be called standard (see [4]). For a standard frame there is a positive invertible adjointable operator $S$, which is called the frame operator, such that the following reconstruction formula holds: $x=\sum_{j} S^{-1}\left(f_{j}\right)\left\langle f_{j}, x\right\rangle_{\mathcal{D}}$, for all $x \in \mathcal{M}$, where the sum converges in norm. Usually, $\left\{S^{-1}\left(f_{j}\right)\right\}_{j}$ is called the dual frame for $\left\{f_{j}\right\}_{j}$. M. Frank and D. Larson also proved that every algebraically finitely or countably generated Hilbert $C^{*}$-module possesses a standard normalized tight frame $([4])$.

Note that in Frank and Larson's papers the first variable in the inner product is linear while in this paper the second one is linear.

The paper is organized as follows: In section 2, we introduce the notion of operator-valued (or $\mathcal{B}$-valued) free Fisher information $\Phi_{\mathcal{B}}^{*}(X: \mathcal{C})$ with respect to a subalgebra $\mathcal{C}$ for a self-adjoint random variable $X$, which is defined in a way that is almost identical to the definition of $\Phi^{*}(X: \mathcal{C})$ given in [20]. Then we show the relations between the operator-valued free Fisher information and the amalgamated freeness. To be precise, we show that $X$ is free from $\mathcal{C}$ with amalgamation over $\mathcal{B}$ if and only if $\Phi_{\mathcal{B}}^{*}(X: \mathcal{C})=\Phi_{\mathcal{B}}^{*}(X: \mathcal{B})$. This result is also a generalization of the corresponding result in [21] but we have to use a quite different method to prove it. In section 3 , we construct the conjugate variable for a semicircular variable $X$ with conditional expectation covariance $E$, and thus we find the Fisher information of such semicircular variables. Then we prove a free relation between $X$ and a subalgebra which has important applications in index theory. It is also shown that $\Phi_{\mathcal{B}}^{*}(X: \mathcal{B})=$ IndexE. At last, D. Shlyakhtenko's free Fisher information with respect to a positive map is considered and we can compute it in some special cases.

\section{Operator-Valued free Fisher information}

The classical Fisher information is derived from the statistical estimation theory which was defined by R. A. Fisher (for some details we refer to [2]). By analogy with the classical case D.Voiculescu introduced the free Fisher information of self-adjoint random variables in a tracial $W *$-noncommutative probability space (see [20], [21]) 
and proved many basic properties. Then A. Nica, D. Shlyakhtenko, and R. Speicher investigated it in view of the cumulant (see [9]) and some minimization problems of it ([10]). D. Shlyakhtenko even introduced the free Fisher information with respect to a positive map ([14]). All of these works are done in the tracial von Neumann algebra framework. Now we generalize the notion of free Fisher information to operator-valued settings, and our work is done in the general von Neumann algebra framework. The way we define it is almost identical to D. Voiculescu's way in [20].

Definition 3. Let $\left(\mathcal{A}, E_{\mathcal{B}}, \mathcal{B}\right)$ be a $\mathcal{B}$-valued $W^{*}$-noncommutative probability space. Suppose $\mathcal{B} \subseteq \mathcal{C} \subseteq \mathcal{A}$ is a von Neumann subalgebra and $X=X^{*} \in \mathcal{A}$ is algebraically free from $\mathcal{C}$ modulo $\mathcal{B} . L_{\mathcal{B}}^{2}(\mathcal{C}[X])$ denotes the Hilbert $\mathcal{B}$-module generated by $\mathcal{C}[X]$ whose inner product is defined by $\langle x, y\rangle:=E_{\mathcal{B}}\left(x^{*} y\right)$, for any $x, y \in \mathcal{C}[X] . \quad \xi \in$ $L_{\mathcal{B}}^{2}(\mathcal{C}[X])$ will be called the conjugate variable of $X$ with respect to $\mathcal{C}$ if

$$
E_{\mathcal{B}}\left(\xi c_{0} X \cdots X c_{n}\right)=\sum_{j=1}^{n} E_{\mathcal{B}}\left(c_{0} X \cdots X c_{j-1}\right) E_{\mathcal{B}}\left(c_{j} X \cdots X c_{n}\right),
$$

for all $c_{0}, c_{1}, \cdots, c_{n} \in \mathcal{C}$.

The free Fisher information of $X$ with respect to $\mathcal{C}$ denoted by $\Phi_{\mathcal{B}}^{*}(X: \mathcal{C})$ is defined by

$$
\Phi_{\mathcal{B}}^{*}(X: \mathcal{C}):=E_{\mathcal{B}}\left(\xi \xi^{*}\right) .
$$

We usually use the notation $\mathcal{J}_{\mathcal{B}}(X: \mathcal{C})$ to denote the conjugate variable of $X$ with respect to $\mathcal{C}$ in a $\mathcal{B}$-valued noncommutative probability space.

Remark. The conjugate variable of $X$ is unique since we always assume $E_{\mathcal{B}}$ to be faithful.

We can also define the conjugate variables $\mathcal{J}_{\mathcal{B}}(X: \mathcal{C})$ in terms of the cumulant function modifying Theorem 4.1 in 9 .

Lemma 4. Let $\left(\mathcal{A}, E_{\mathcal{B}}, \mathcal{B}\right)$ be a $\mathcal{B}$-valued noncommutative probability space. Suppose $\mathcal{B} \subseteq \mathcal{C} \subseteq \mathcal{A}$ is a von Neumann subalgebra and $X=X^{*} \in \mathcal{A}$ is algebraically free from $\mathcal{C}$ modulo $\mathcal{B}$. Let $E_{\mathcal{C}}$ be a conditional expectation of $\mathcal{A}$ onto $\mathcal{C}$ such that $E_{\mathcal{B}}=E_{\mathcal{B}} E_{\mathcal{C}} . \xi \in L_{\mathcal{B}}^{2}(\mathcal{C}[X])$ is the conjugate variable of $X$ with respect to $\mathcal{C}$ if and only if the following equations hold:

$$
\begin{cases}k_{\mathcal{C}}^{(1)}(\xi c)=0, & \text { for any } c \in \mathcal{C} \\ k_{\mathcal{C}}^{(2)}(\xi \otimes c a)=\delta_{a X} E_{\mathcal{B}}(c), & \text { for any } c \in \mathcal{C} \\ k_{\mathcal{C}}^{(m+1)}\left(\xi \otimes c_{1} a_{1} \otimes \cdots \otimes c_{m} a_{m}\right)=0, & \text { for any } c_{1}, \cdots, c_{m} \in \mathcal{C} ; m \geq 2\end{cases}
$$

where $a_{1}, \cdots, a_{m} \in\{X\} \cup \mathcal{C}$, and $k_{\mathcal{C}}$ is the cumulant function induced by $E_{\mathcal{C}}$.

Proof. Since $E_{\mathcal{B}}$ is faithful and $E_{\mathcal{B}}=E_{\mathcal{B}} E_{\mathcal{C}}$ we know equation (1) is equivalent to

$$
E_{\mathcal{C}}\left(\xi c_{0} X \cdots X c_{n}\right)=\sum_{j=1}^{n} E_{\mathcal{B}}\left(c_{0} X \cdots X c_{j-1}\right) E_{\mathcal{C}}\left(c_{j} X \cdots X c_{n}\right) .
$$

Now suppose $\xi$ is the conjugate variable of $X$. Then

$$
k_{\mathcal{C}}^{(1)}(\xi c)=E_{\mathcal{C}}^{(1)}(\xi c)=0, \text { for all } c \in \mathcal{C},
$$

and

$$
\begin{aligned}
k_{\mathcal{C}}^{(2)}(\xi \otimes c a) & =E_{\mathcal{C}}^{(2)}(\xi \otimes c a)-k_{\mathcal{C}}^{(1)}\left(\xi E_{\mathcal{C}}(c a)\right) \\
& =E_{\mathcal{C}}(\xi c a)=\delta_{a X} E_{\mathcal{B}}(c) .
\end{aligned}
$$


To prove the third equation we proceed by induction on $m \geq 2$. When $m=2$, we have

$$
\begin{aligned}
& E_{\mathcal{C}}^{(3)}\left(\xi \otimes c_{1} a_{1} \otimes c_{2} a_{2}\right) \\
&=k_{\mathcal{C}}^{(1)}\left(\xi E_{\mathcal{C}}^{(2)}\left(c_{1} a_{1} \otimes c_{2} a_{2}\right)\right)+k_{\mathcal{C}}^{(2)}\left(\xi \otimes c_{1} a_{1} E_{\mathcal{B}}\left(c_{2} a_{2}\right)\right) \\
&+k_{\mathcal{C}}^{(2)}\left(\xi E_{\mathcal{C}}\left(c_{1} a_{1}\right) \otimes c_{2} a_{2}\right)+k_{\mathcal{C}}^{(3)}\left(\xi \otimes c_{1} a_{1} \otimes c_{2} a_{2}\right) \\
&= \delta_{a_{1} X} E_{\mathcal{B}}\left(c_{1}\right) E_{\mathcal{C}}\left(c_{2} a_{2}\right)+\delta_{a_{2} X} E_{\mathcal{B}}\left(c_{1} a_{1}\right) E_{\mathcal{C}}\left(c_{2}\right) \\
&+k_{\mathcal{C}}^{(3)}\left(\xi \otimes c_{1} a_{1} \otimes c_{2} a_{2}\right) .
\end{aligned}
$$

Noting that $\xi$ is the conjugate variable, so we get

$$
k_{\mathcal{C}}^{(3)}\left(\xi \otimes c_{1} a_{1} \otimes c_{2} a_{2}\right)=0 .
$$

Now assuming the equation holds for the numbers $\leq m-1$, then

$$
\begin{aligned}
& E_{\mathcal{C}}\left(\xi \otimes c_{1} a_{1} \otimes \cdots \otimes c_{m} a_{m}\right) \\
& \quad=\sum_{1 \leq j \leq m+1} k_{\mathcal{C}}^{(2)}\left(\xi E_{\mathcal{C}}^{(j-1)}\left(c_{1} a_{1} \otimes \cdots \otimes c_{j-1} a_{j-1}\right)\right. \\
& \left.\quad \otimes c_{j} a_{j} E_{\mathcal{C}}^{(m-j)}\left(c_{j+1} a_{j+1} \otimes \cdots \otimes c_{m} a_{m}\right)\right)+k_{\mathcal{C}}^{(m+1)}\left(\xi \otimes c_{1} a_{1} \otimes \cdots \otimes c_{m} a_{m}\right) .
\end{aligned}
$$

Since $\xi$ is the conjugate system of $X$ and because of (3), we have

$$
k_{\mathcal{C}}^{(m+1)}\left(\xi \otimes c_{1} a_{1} \otimes \cdots \otimes c_{m} a_{m}\right)=0 .
$$

It is easy to see that the above process is invertible and so the converse is also true.

Now we can prove the following proposition using the above result.

Lemma 5. With notation as in the above lemma, suppose $\mathcal{B}[X]$ is free from $\mathcal{C}$ with amalgamation over $\mathcal{B}$. Then $\mathcal{J}_{\mathcal{B}}(X: \mathcal{B})=\mathcal{J}_{\mathcal{B}}(X: \mathcal{C})$.

Proof. Since $\mathcal{J}_{\mathcal{B}}(X: \mathcal{B})$ is the conjugate variable of $X$ with respect to $\mathcal{B}$ and $\mathcal{B}[X]$ is free from $\mathcal{C}$, we have for any $c \in \mathcal{C}$,

$$
\begin{aligned}
E_{\mathcal{B}} & \left.E_{\mathcal{C}}\left(\mathcal{J}_{\mathcal{B}}(X: \mathcal{B}) c\right)\right) \\
& =E_{\mathcal{B}} E_{\mathcal{C}}^{(2)}\left(\mathcal{J}_{\mathcal{B}}(X: \mathcal{B}) \otimes c\right) \\
& =E_{\mathcal{B}}\left(k_{\mathcal{C}}^{(2)}\left(\mathcal{J}_{\mathcal{B}}(X: \mathcal{B}) \otimes c\right)+k_{\mathcal{C}}^{(1)}\left(\mathcal{J}_{\mathcal{B}}(X: \mathcal{B}) c\right)\right) \\
& =E_{\mathcal{B}}\left(\mathcal{J}_{\mathcal{B}}(X: \mathcal{B}) E_{\mathcal{B}}(c)\right) \\
& =0 .
\end{aligned}
$$

The second equality follows from Lemma 2. Since $E_{\mathcal{B}}$ is faithful, we infer that $k_{\mathcal{C}}^{(1)}\left(\mathcal{J}_{\mathcal{B}}(X: \mathcal{B})\right)=0$. Then

$$
\begin{aligned}
k_{\mathcal{C}}^{(2)}( & \left.\mathcal{J}_{\mathcal{B}}(X: \mathcal{B}) \otimes e X\right) \\
= & k_{\mathcal{C}}^{(3)}\left(\mathcal{J}_{\mathcal{B}}(X: \mathcal{C}) \otimes e \otimes X\right) \\
& +k_{\mathcal{C}}^{(2)}\left(k_{\mathcal{C}}^{(2)}\left(\mathcal{J}_{\mathcal{B}}(X: \mathcal{B}) \otimes e\right) k_{\mathcal{C}}(X)\right)+k_{\mathcal{C}}^{(2)}\left(\left(\mathcal{J}_{\mathcal{B}}(X: \mathcal{B}) k_{\mathcal{C}}(e) \otimes X\right)\right. \\
= & k_{\mathcal{D}}^{(2)}\left(\mathcal{J}_{\mathcal{B}}(X: \mathcal{B}) E_{\mathcal{B}}(e) \otimes X\right)=E_{\mathcal{B}}(e)
\end{aligned}
$$

where $e \in \mathcal{C}$. 
For all $m \geq 2 ; e_{1}, e_{2}, \cdots, e_{m} \in \mathcal{C}$, we have

$$
\begin{aligned}
k_{\mathcal{C}}^{(m+1)} & \left(\mathcal{J}_{\mathcal{B}}(X: \mathcal{B}) \otimes e_{1} X \otimes \cdots \otimes e_{m} X\right) \\
& =k_{\mathcal{B}}^{(m+1)}\left(\mathcal{J}_{\mathcal{B}}(X: \mathcal{B}) \otimes E_{\mathcal{B}}\left(e_{1}\right) X \otimes \cdots \otimes E_{\mathcal{B}}\left(e_{m}\right) X\right) \\
& =0 .
\end{aligned}
$$

Thus it follows from Lemma 4 that $\mathcal{J}_{\mathcal{B}}(X: \mathcal{B})=\mathcal{J}_{\mathcal{B}}(X: \mathcal{C})$ since $\mathcal{J}_{\mathcal{B}}(X: \mathcal{C})$ is unique.

Remark. The above proposition is a generalization of Proposition 3.6 in [20]. Obviously we have not used the method of proof explained in [20] since $E_{\mathcal{B}}$ is not tracial in general.

In the proof of the following theorem we will use the notion of the $\mathcal{B}$-valued liberation gradient $([21], 9])$. Let $\mathcal{B} \subseteq \mathcal{C}_{1}, \mathcal{C}_{2} \subseteq \mathcal{A}$ be subalgebras of $\mathcal{A}$, which are algebraically free modulo $\mathcal{B}$. We define a derivation

$$
\delta_{\mathcal{C}_{1}: \mathcal{C}_{2}}: \mathcal{C}_{1} \vee \mathcal{C}_{2} \longrightarrow\left(\mathcal{C}_{1} \vee \mathcal{C}_{2}\right) \otimes\left(\mathcal{C}_{1} \vee \mathcal{C}_{2}\right)
$$

as follows:

$$
\delta_{\mathcal{C}_{1}: \mathcal{C}_{2}}(c)= \begin{cases}c \otimes_{\mathcal{B}} 1-1 \otimes_{\mathcal{B}} c, & \text { if } c \in \mathcal{C}_{1}, \\ 0, & \text { if } c \in \mathcal{C}_{2} .\end{cases}
$$

$\xi \in L_{D}^{2}\left(\mathcal{C}_{1} \vee \mathcal{C}_{2}\right)$ will be called the $\mathcal{B}$-liberation gradient for $\left(\mathcal{C}_{1}, \mathcal{C}_{2}\right)$ if it satisfies

$$
E_{\mathcal{B}}(\xi m)=\left(E_{\mathcal{B}} \otimes E_{\mathcal{B}}\right)\left(\delta_{\mathcal{C}_{1}: \mathcal{C}_{2}} m\right)
$$

for any $m \in\left(\mathcal{C}_{1} \vee \mathcal{C}_{2}\right)$. We denote it by $j_{\mathcal{B}}\left(\mathcal{C}_{1}: \mathcal{C}_{2}\right)$. An important property of the liberation gradient is: $\mathcal{C}_{1}, \mathcal{C}_{2}$ is $\mathcal{B}$-free $\Leftrightarrow j_{\mathcal{B}}\left(\mathcal{C}_{1}: \mathcal{C}_{2}\right)=0([9])$.

Theorem 6. (i) If $\mathcal{C}$ is free from $\mathcal{B}[X]$ with amalgamation over $\mathcal{B}$, and if $\Phi_{\mathcal{B}}^{*}(X: \mathcal{B})$ exists, then $\Phi_{\mathcal{B}}^{*}(X: \mathcal{C})$ exists and $\Phi_{\mathcal{B}}^{*}(X: \mathcal{C})=\Phi_{\mathcal{B}}^{*}(X: \mathcal{B})$.

(ii) Suppose $\Phi_{\mathcal{B}}^{*}(X: \mathcal{B}), \Phi_{\mathcal{B}}^{*}(X: \mathcal{C})$ exist and are equal to each other. Then $X$ is free from $\mathcal{C}$ with amalgamation over $\mathcal{B}$.

Proof. (i) can be inferred from Lemma 5 directly.

(ii) We can prove this following D. Voiculescu's method in [21. Here we sketch it briefly. Since $E_{\mathcal{B}[X]} E_{\mathcal{B}}=E_{\mathcal{B}}$ and $E_{\mathcal{B}[X]}$ is self-adjoint, we have

$$
\mathcal{J}_{\mathcal{B}}(X: \mathcal{B})=E_{\mathcal{B}} \mathcal{J}_{\mathcal{B}}(X: \mathcal{C})
$$

and since $\Phi_{\mathcal{B}}^{*}(X: \mathcal{C})=\Phi_{\mathcal{B}}^{*}(X: \mathcal{B})$, we get

$$
E_{\mathcal{B}}\left(\mathcal{J}_{\mathcal{B}}(X: \mathcal{B}) \mathcal{J}_{\mathcal{B}}(X: \mathcal{B})^{*}\right)=E_{\mathcal{B}}\left(\mathcal{J}_{\mathcal{B}}(X: \mathcal{C}) \mathcal{J}_{\mathcal{B}}(X: \mathcal{C})^{*}\right)
$$

and $\mathcal{J}_{\mathcal{B}}(X: \mathcal{B})=\mathcal{J}_{\mathcal{B}}(X: \mathcal{C})$

Therefore,

$$
\begin{aligned}
& j(\mathcal{B}[X]: \mathcal{C})=\left[\mathcal{J}_{\mathcal{B}}(X: \mathcal{C}), \mathcal{B}[X]\right] \\
& \quad=\left[\mathcal{J}_{\mathcal{B}}(X: \mathcal{B}), \mathcal{B}[X]\right] \in L_{\mathcal{B}}^{2}(\mathcal{B}[X])
\end{aligned}
$$

where $j$ is the $\mathcal{B}$-liberation gradient. Hence, $j(\mathcal{B}[X]: \mathcal{C}) \in L_{\mathcal{B}}^{2}(\mathcal{B}[X])$ and $j(\mathcal{B}[X]: \mathcal{B})$ $=0$ since $\langle j(\mathcal{B}[X]: \mathcal{C}), \mathcal{B}[X]\rangle_{\mathcal{B}}=0$. Thus $j(\mathcal{B}[X]: \mathcal{C})=0$, and the desired result is obtained. 


\section{Operator-VALUED FREE Fisher INFORMATION OF SEMICIRCULAR VARIABLES}

Recall that the most important random variable is the semicircular variable. In an operator-valued noncommutative probability space, a semicircular variable is connected with a linear map.

Definition $7\left([13,[18])\right.$. Let $\left(\mathcal{A}, E_{\mathcal{B}}, \mathcal{B}\right)$ be a noncommutative probability space and let $\eta: \mathcal{B} \rightarrow \mathcal{B}$ be a linear map. A self-adjoint element $X \in \mathcal{A}$ will be called a semicircular variable with covariance $\eta$ (or $\eta$-semicircular variable) if it satisfies $k_{\mathcal{B}}^{(1)}(X)=0, k_{\mathcal{B}}^{(2)}(X \otimes b X)=\eta(b), k_{\mathcal{B}}^{(m+1)}\left(X \otimes b_{1} X \otimes \cdots \otimes b_{m} X\right)=0$, for all $b, b_{1}, \cdots, b_{m} \in \mathcal{B}$.

In general, the construction of conjugate variables is difficult, but for a semicircular variable with the conditional expectation covariance we can do it.

Given a conditional expectation $E$ of $\mathcal{B}$ onto $\mathcal{D}$, we can define a $\mathcal{D}$-valued inner product $\langle x, y\rangle_{\mathcal{D}}:=E\left(x^{*} y\right)$, for all $x, y \in \mathcal{B}$, just as in section 1 , and use $L_{\mathcal{D}}^{2}(\mathcal{B})$ to denote the completion of $\mathcal{B}$ with respect to the norm $\|x\|:=\left\|\langle x, x\rangle_{\mathcal{D}}\right\|^{\frac{1}{2}}$.

Proposition 8. Let $\mathcal{D} \subseteq \mathcal{B} \subseteq \mathcal{A}$ be a von Neumann subalgebras' inclusion. $E$ : $\mathcal{B} \rightarrow \mathcal{D}, E_{\mathcal{B}}: \mathcal{A} \rightarrow \mathcal{B}$ are conditional expectations, and suppose $E_{\mathcal{D}}=E_{\mathcal{B}}, L_{\mathcal{D}}^{2}(\mathcal{B})$ $=\mathcal{B}$. Let $\left\{f_{i}\right\} \in L_{\mathcal{D}}^{2}(\mathcal{B})$ be a standard frame in $L_{\mathcal{D}}^{2}(\mathcal{B})$. Let $X=X^{*} \in\left(\mathcal{A}, E_{\mathcal{B}}, \mathcal{B}\right)$ be an E-semicircular variable. Then

$$
\begin{aligned}
& \mathcal{J}_{\mathcal{D}}(X: \mathcal{B})=\sum_{i} g_{i} X E\left(f_{i}^{*}\right), \\
& \mathcal{J}_{\mathcal{D}}(X: \mathcal{D})=X \\
& \mathcal{J}_{\mathcal{B}}(X: \mathcal{B})=\sum_{i} g_{i} X f_{i}^{*}
\end{aligned}
$$

where $\left\{g_{i}\right\}$ is a dual frame for $\left\{f_{i}\right\}$ and the above infinite sums are assumed to be convergent in norm.

Proof. To prove the first equality we need to verify that $\sum g_{i} X E\left(f_{i}^{*}\right)$ satisfies the equalities in Lemma 4. In fact: $k_{\mathcal{B}}^{(1)}\left(\sum g_{i} X E\left(f_{i}^{*}\right)\right)=0$, since $X$ is semicircular, and

$$
\begin{aligned}
k_{\mathcal{B}}^{(2)}( & \left.\sum g_{i} X E\left(f_{i}^{*}\right) \otimes b X\right) \\
& =E_{\mathcal{B}}\left(\sum g_{i} X E\left(f_{i}^{*}\right) b X\right) \\
& =\sum g_{i} E\left(f_{i}^{*} E(b)\right) \quad \text { (since } X \text { is } E \text {-semicircular) } \\
& =E(b) \quad\left(\text { since }\left\{g_{i}\right\} \text { is the dual frame of }\left\{f_{i}\right\}\right)
\end{aligned}
$$

and for all $m \geq 2$,

$$
k_{\mathcal{B}}^{(m+1)}\left(\sum g_{i} X E\left(f_{i}^{*}\right) \otimes b_{1} X \otimes \cdots \otimes b_{m} X\right)=0 \quad \text { (since } X \text { is semicircular). }
$$

The second equality can be proved in a way similar to the above by noting that $X \in\left(\mathcal{A}, E_{\mathcal{D}}, \mathcal{D}\right)$ is an $i d$-semicircular variable.

The third one can also be obtained in the same way.

Theorem 9. Let the notation and conditions be as in the above proposition. Then $\Phi_{\mathcal{D}}^{*}(X: \mathcal{B})=\sum_{j} E\left(f_{j}\right) E\left(g_{j}^{*}\right)=1 ; \Phi_{\mathcal{D}}^{*}(X: \mathcal{D})=1 ; \Phi_{\mathcal{B}}^{*}(X: \mathcal{B})=\sum_{i} f_{i} g_{i}^{*}$. 
Proof. We only prove the first one. In fact

$$
\begin{aligned}
\Phi_{\mathcal{D}}^{*} & (X: \mathcal{B})=E_{\mathcal{D}}\left(\mathcal{J}_{\mathcal{D}}(X: \mathcal{B}) \mathcal{J}_{\mathcal{D}}(X: \mathcal{B})^{*}\right) \\
& =E_{\mathcal{D}}\left(\sum_{i} g_{i} X E\left(f_{i}^{*}\right) \sum_{j} E\left(f_{j}\right) X g_{j}^{*}\right) \\
& =E\left(\sum_{i, j} g_{i} E_{\mathcal{B}}\left(X E\left(f_{i}^{*}\right) E\left(f_{j}\right) X\right) g_{j}^{*}\right) \\
& =E\left(\sum_{i, j} g_{i} E\left(E\left(f_{i}^{*}\right) E\left(f_{j}\right)\right) g_{j}^{*}\right) \\
& =E\left(\sum_{j} E\left(f_{j}\right) g_{j}^{*}\right)=\sum_{j} E\left(f_{j}\right) E\left(g_{j}^{*}\right) \\
& =1 .
\end{aligned}
$$

The remaining two equalities can be proved in a similar way.

Theorem 10. Let $X=X^{*} \in\left(\mathcal{A}, E_{\mathcal{B}}, \mathcal{B}\right)$ be an E-semicircular variable, where $E$ is the conditional expectation of $\mathcal{B}$ onto $\mathcal{D}$ and assume the Hilbert $\mathcal{D}$-module $L_{\mathcal{D}}^{2}(\mathcal{B})=\mathcal{B}$ to be finitely or countably generated. Let $E_{\mathcal{D}}:=E E_{\mathcal{B}}$. Then $X$ is free from $\mathcal{B}$ with amalgamation over $\mathcal{D}$ in the $\mathcal{D}$-valued noncommutative probability $\operatorname{space}\left(\mathcal{A}, E_{\mathcal{D}}, \mathcal{D}\right)$.

Proof. From Theorem $9, \Phi_{\mathcal{D}}^{*}(X: \mathcal{B})=\sum_{j} E\left(f_{j}\right) E\left(g_{j}^{*}\right)=1$ and $\Phi_{\mathcal{D}}^{*}(X: \mathcal{D})=1$, and from Theorem 6 we get the desired result.

The above theorem has an important application: We use $W^{*}\langle\mathcal{B}, X\rangle$ to denote the von Neumann algebra generated by $\mathcal{B}$ and $X$. Then $W^{*}\langle\mathcal{B}, X\rangle \cong \mathcal{B} *_{\mathcal{D}} L^{\infty}[0,1]$, which is a construction frequently used in index theory (see [11]), where $*_{\mathcal{D}}$ denotes the free product amalgamated with $\mathcal{D}$.

After the introduction of Jones' paper [7] several notions of index for conditional expectations between von Neumann algebras appeared (see [1], [12], 16]). This generalization forces the index to be no more a scalar but a positive operator. A conditional expectation $E: \mathcal{M} \rightarrow \mathcal{N}$ is said to be of finite index if there exists an $\mathcal{N}$-orthonormal basis $\left\{m_{i}\right\}_{i \in I}$ of the $\mathcal{N}$-Hilbert module $\mathcal{M}$ such that $\sum m_{i} m_{i}^{*}$ is weakly convergent and IndexE $:=\sum_{i} m_{i} m_{i}^{*}([12])$. Since an orthonormal basis is a special normalized tight frame, we get the following.

Corollary 11. Let $E: \mathcal{B} \rightarrow \mathcal{D}$ be a conditional expectation. If $L_{\mathcal{D}}^{2}(\mathcal{B})=\mathcal{B}$, then $\Phi_{\mathcal{B}}^{*}(X: \mathcal{B})=$ IndexE.

\section{Free Fisher information With Respect to a Positive map}

In [14, D. Shlyakhtenko introduced the free Fisher information with respect to a positive map in a tracial $W *$-noncommutative probability $(\mathcal{A}, \tau)$, where $\tau$ is the tracial state on $\mathcal{A}$, as follows.

Definition $12\left([14)\right.$. Suppose $(\mathcal{A}, \tau)$ is a $W^{*}$ probability space with $\tau$ faithful trace and $X=X^{*} \in \mathcal{A}$. Let $\mathcal{B} \subset \mathcal{A}$ be a unital $W^{*}$ subalgebra, and let $\eta: \mathcal{B} \rightarrow \mathcal{B}$ be a (completely) positive map. $\mathcal{J}(X: \mathcal{B}, \eta) \in L^{2}(\mathcal{B}[X])$ will be called the conjugate 
variable for $X$ with respect to $\mathcal{B}$ and $\eta$ if it satisfies

$$
\tau\left(\mathcal{J}(X: \mathcal{B}, \eta) b_{0} X b_{1} X \cdots X b_{n}\right)=\sum_{j=1}^{n} \tau\left(\eta\left(E_{\mathcal{B}}\left(b_{0} X \cdots b_{j-1}\right)\right) b_{j} X \cdots X b_{n}\right),
$$

for all $b_{0}, b_{1}, \cdots, b_{n} \in \mathcal{B}$, where $E_{\mathcal{B}}$ is the unique conditional expectation of $\mathcal{A}$ onto $\mathcal{B}$ such that $\tau E_{\mathcal{B}}=\tau$. The free Fisher information of $X$ with respect to $\mathcal{B}$ and $\eta$ is defined by

$$
\Phi^{*}(X: \mathcal{B}, \eta):=\tau\left(\mathcal{J}(X: \mathcal{B}, \eta) \mathcal{J}(X: \mathcal{B}, \eta)^{*}\right) .
$$

D. Shlyakhtenko extended many facts proven in $\left[20\right.$ to $\Phi^{*}(., \mathcal{B}, \eta)$ with similar proofs, and he also found out the relation between the Jones' index for $I I_{1}$ subfactors and the free Fisher information with respect to a map. We can see that in the tracial case D. Shlyakhtenko's notion is similar to ours to some extent. In addition, we can also compute the free Fisher information with respect to a modular frame operator.

Theorem 13. Let $\mathcal{D} \subseteq \mathcal{B} \subseteq(\mathcal{A}, \tau)$ be a von Neumann subalgebras' inclusion, and let $E: \mathcal{B} \rightarrow \mathcal{D}$ be a conditional expectation. Assume $\eta$ is a (completely) positive invertible map of $\mathcal{B}$ onto $\mathcal{B}$, and let $X$ be a semicircular variable with covariance E. Then

$$
\mathcal{J}(X: \mathcal{B}, \eta)=\sum_{i \in \mathbb{N}} f_{i} X f_{i}^{*}, \quad \Phi^{*}(X: \mathcal{B}, \eta)=\sum_{i} \tau\left(\eta\left(f_{i}\right) f_{i}^{*}\right),
$$

where $\left\{f_{i}\right\}$ is a frame in the Hilbert $\mathcal{D}$-module $L_{\mathcal{D}}^{2}(\mathcal{B})$ with frame operator $\eta$, such that the above sums are convergent.

Proof. To prove $\mathcal{J}(X: \mathcal{B}, \eta)=\sum_{i \in \mathbb{N}} f_{i} X f_{i}^{*}$, from [14], Proposition 6.2, we only need to show that

$$
\tau\left(\sum f_{i} X f_{i}^{*} b X b^{\prime}\right)=\tau\left(\eta(b) b^{\prime}\right)
$$

for all $b, b^{\prime} \in \mathcal{B}$. In fact,

$$
\begin{gathered}
\tau\left(\sum f_{i} X f_{i}^{*} b X b^{\prime}\right)=\tau\left(E_{\mathcal{B}}\left(\sum f_{i} X f_{i}^{*} b X\right) b^{\prime}\right) \\
=\tau\left(\sum f_{i} E_{\mathcal{B}}\left(X f_{i}^{*} b X\right) b^{\prime}\right)=\tau\left(\sum f_{i} E\left(f_{i}^{*} b\right) b^{\prime}\right) \\
=\tau\left(\sum f_{i} E\left(f_{i}^{*} \eta^{-1} \eta(b)\right) b^{\prime}\right)=\tau\left(\eta(b) b^{\prime}\right) .
\end{gathered}
$$

Hence we obtain (5). Then

$$
\begin{aligned}
\Phi^{*}(X & : \mathcal{B}, \eta)=\tau\left(\left(\sum_{i} f_{i} X f_{i}^{*}\right)\left(\sum_{j} f_{j} X f_{j}^{*}\right)\right) \\
& =\tau\left(\sum_{i, j} f_{i} E_{\mathcal{B}}\left(X f_{i}^{*} f_{j} X\right) f_{j}^{*}\right) \\
& =\tau\left(\sum_{i, j} f_{i} E\left(f_{i}^{*} f_{j}\right) f_{j}^{*}\right)=\tau\left(\sum_{j} \eta\left(f_{j}\right) f_{j}^{*}\right) .
\end{aligned}
$$

\section{ACKNOWLEDGMENT}

The authors would like to express their deep gratitude for Prof. David Larson's and the referee's important advice. 


\section{REFERENCES}

1. M. Baillet, Y. Denizeau and J. -F.Havet, Indice d'une espérance conditionelle. Compositio Math. 66 (1988) 199-236. MR0945550 (90e:46050)

2. T. M. Cover and Joy A. Thomas, Elements of information theory. John Wiley \& Sons, Inc. Chichester, 1976. MR1122806 (92g:94001)

3. M. Frank, E. Kirchberg, On conditional expectations of finite index J. Operator Theory , 40 (1998), 87-111. MR 1642530 (2000k:46080)

4. M. Frank, D. R. Larson, A module frame concept for Hilbert $C^{*}$-modules. in: Functional and Harmonic Analysis of Wavelets (San Antonio, TX, Jan. 1999), A.M.S., Providence, R.I., Contemp. Math. 247 (2000), 207-233. MR 1738091 (2001b:46094)

5. M. Frank, D. R. Larson, Frames in Hilbert $C^{*}$-modules and $C^{*}$-algebras. J. Operator Theory, 48 (2002), 273-314. MR.1938798 (2003i:42040)

6. L. Ge, Applications of free entropy to finite von Neumann algebras. II Annals of Mathematics, 147(1998), 143-157. MF,1609522 (99c:46068)

7. V. F. Jones, Index for subfactors. Invent. Math. 72 (1983), 1-25. MR0696688 (84d:46097)

8. C. Lance, Hilbert $C^{*}-$ Modules. LMS note series, 210, Cambridge University Press, 1995. MR1325694 (96k:46100)

9. A. Nica, D.Shlyakhtenko, R.Speicher, Operator-valued distributions. 1. Characterizations of freeness. IMRN 2002: 29 (2002) 1509-1538. MR.1907203 (2003f:46105)

10. A. Nica, D. Shlyakhtenko, R. Speicher, Some minimization problems for the free analogue of the Fisher information. Advances in Mathematics 141 (1999) 282-321. MR1671766 (2000b:46120)

11. S. Popa, Markov traces on universal Jones algebras and subfactors of finite index. Invent.Math 111 (1993), 375-405. MR 1198815 (94c:46128)

12. S. Popa, Classsification of subfactors and their endomorphisms. CMBS 86 AMS (1995). MR 1339767 (96d:46085)

13. R. Speicher, Combinatorial theory of the free product with amalgamation and operator-valued free probability theory. Memoirs of AMS 627 (1998). MF.1407898 (98i:46071)

14. D. Shlyakhtenko, Free entropy with respect to a completely positive map. Amer. J. Math 122 (2000), 45-81. MR1737257 (2001f:46102)

15. V. S. Sunder, An invitation to von Neumann algebras. Springer-Verlag, New York, 1987. MR0866671 (88f:46115)

16. Y. Watatani, Index for $C^{*}$-subalgebras. Memoirs AMS vol. 83, 424 (1990). MR0996807 (90i:46104)

17. D. Voiculescu, K. J. Dykema, A. Nica, Free random variables. CRM Monograph Series, Vol. 1, AMS, 1992. MR.1217253 (94c:46133)

18. D. Voiculescu, Operations on certain Non-commutative operator-valued random variables. Astérisque 232 (1995), 243-275. MR1372537 (97b:46081)

19. D. Voiculescu, The analogues of entropy and of Fisher's information measure in free probability theory III: The absence of Cartan subalgebras, Geometric and Functional Analysis. Vol.6. 1(1996). MR.1371236 (96m:46119)

20. D. Voiculescu, The analogues of entropy and of Fisher's information measure in free probability theory V. Noncommutative Hibert Transforms. Inventiones Mathematicae, 132(1998), 189-227. MR1618636 (99d:46087)

21. D. Voiculescu, The analogues of entropy and of Fisher's information measure in free probability theory VI. Liberation and mutual free information. Advances in Mathematics 146(1999), 101-166. MR 1711843(2001a:46064)

Lmam, School of Mathematical Sciences, Peking University, Beijing 100871, PeoPLE'S Republic of China

Current address: College of Science, Nanjing University of Aeronautics and Astronautics, Nanjing 210016, People's Republic of China

E-mail address: b.meng@nuaa.edu.cn

LMam, School of Mathematical Sciences, Peking University, Beijing 100871, PeoPLE'S RePUBLIC OF CHINA

Lmam, School of Mathematical Sciences, Peking University, Beijing 100871, PeoPLE'S RePUBlic OF CHINA 